\title{
Hardware-in-the-Loop Emulation of Three-Phase Grid Impedance for characterizing Impedance-Based Instability
}

\author{
Tuomas Messo $^{1}$ Jussi Sihvo $^{1}$ Tomi Roinila ${ }^{2}$ Tommi Reinikka ${ }^{2}$ Roni Luhtala ${ }^{2}$ \\ ${ }^{1}$ Department of Electrical Engineering, Tampere University of Technology, Finland, \{tuomas.messo\} @tut.fi \\ ${ }^{2}$ Department of Automation Sciences and Engineering, Tampere University of Technology, Finland
}

\begin{abstract}
The amount of grid-connected power electronic converters is increasing as the world's energy production shifts toward sustainable sources. Poor power quality and harmonic resonances have been reported which have been shown to be caused by grid-connected converters. Accurate modeling tools are required to characterize the conditions for instability and to design stable power-electronicsbased power systems.

Unstable behavior can be identified by using models implemented in circuit simulators or using powerhardware-in-the-loop setups. The unstable resonance occurs when inverter control system interacts with the grid impedance. However, a very wide impedance-bank is required in the laboratory to test inverter stability when grid impedance is expected to vary significantly. Moreover, stability tests are often limited to cases where grid impedance is approximated as an inductance. This paper proposes a method for emulating the grid impedance in a hardware-in-the-loop setup which eliminates the need for bulky passive components and allows arbitrary grid impedance to be emulated. As a result, the inverter can be tested with a varying grid impedance to determine the exact conditions for unstable behavior. Moreover, the grid impedance can be changed online to emulate the behavior of a time-varying power grid in real time.
\end{abstract}

Keywords: hardware-in-the-loop, grid impedance, emulation

\section{Introduction}

The amount of grid-connected power electronic converters, such as wind and solar inverters is rapidly increasing as the amount of renewable power generation is ramped up (Bose, 2013). Stability of conventional power systems has been mainly determined by power balance, i.e., the produced power has to match the load power to keep grid frequency and amplitude within acceptable limits. However, the stability of modern power systems is challenged by the dynamic behavior of grid-connected power electronic converters, and especially, their small-signal characteristics (Wang et al., 2014; Messo et al., 2013).

Grid-connected converters have been shown to introduce stability issues when the penetration of renewables is significant (Enslin and Heskes, 2004). This has been

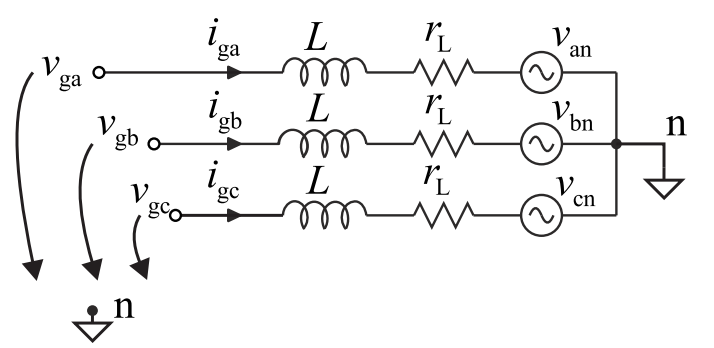

Figure 1. Three-phase grid impedance.

shown to be mainly caused by poorly damped resonance formed together by inverter output impedance and the grid impedance (Sun, 2011; Wen et al., 2016; Suntio et al., 2017). Wind power inverters were found out to cause instability when connected to a series-compensated line (Belkin, 2010) and photovoltaic inverters have been reported to become unstable in a grid that has high inductance (Yang et al., 2014). Instability and resonance issues can be prevented if the inverter impedance is designed to have larger impedance than the grid or if both impedances resemble passive circuits (Harnefors et al., 2016).

Immunity of a grid-connected inverter to impedancebased instability can be evaluated by connecting the inverter to the power system through an inductance which has sufficiently large value. A weak grid is usually approximated by a large inductance or combination of resistive and inductive elements (Gavrilovic, 1991). However, in reality the grid impedance can be hardly described as a lossy inductor since it may exhibit resonances (Jessen et al., 2015). In practice this means that one should have sufficient physical impedance-bank in the laboratory to test converters in different grid conditions. An interesting alternative is to use a real-time simulator paired with a linear amplifier to emulate the grid impedance which gives more freedom to define the nature of grid impedance, e.g., a series or parallel resonance. Promising results have been presented in the literature, such as in (Kotsampopoulos et al., 2015) where a real-time simulator was used to emulate part of the grid impedance in a hardware-in-the-loop simulation. However, the performance of the impedance emulation method was not validated by measuring the emulated impedance.

This paper proposes a method to emulate the grid 


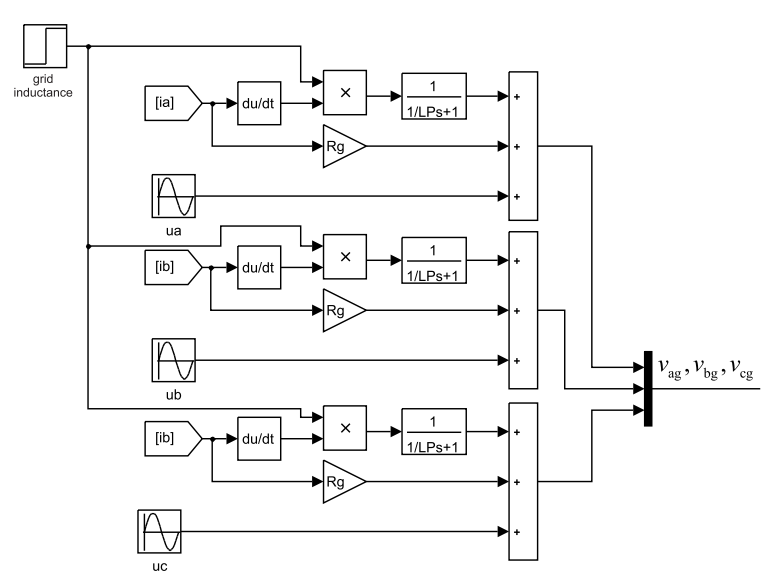

Figure 2. Simulink model to realize the grid impedance in Figure 1.

impedance behavior using power-hardware-in-the-loop (PHIL) setup, which can be used in characterizing impedance-based stability of grid-connected inverters. The method enables modifying the grid impedance online allowing stability studies of grid-connected converters in time-varying grid conditions. The limitations of PHILimplementation due to sampling delay are also discussed. The delay is shown to introduce a considerable error in the phase of the emulated grid impedance.

The paper is organized as follows: Section II explains how a three-phase grid impedance is modeled in the MATLAB Simulink environment. Such model can be directly build into $\mathrm{C}$-code and ran on a real-time simulator. Section III shows a simulation case where grid inductance is stepped up to destabilize a grid-connected inverter. The practical implementation of the grid impedance emulator and its limitations are discussed in Section IV where a dSPACE real-time simulator and a linear amplifier are used to emulate an inductive grid impedance. Final conclusions are summarized in Section V.

\section{Grid impedance model}

Circuit diagram of a three-phase grid impedance is as depicted in Figure 1 where $v_{\mathrm{an}}, v_{\mathrm{bn}}$ and $v_{\mathrm{cn}}$ represent the ideal grid voltages. The grid is formed by three identical branches which have resistance and inductance. Grid voltages seen by a grid-connected inverter, i.e., $v_{\text {ga }}, v_{\text {gb }}$ and $v_{\mathrm{gc}}$ at the PCC, can be solved by utilizing basic circuit theory and given according to (1).

$$
\begin{aligned}
& v_{\mathrm{ga}}(t)=L \frac{\mathrm{d}}{\mathrm{d} t} i_{\mathrm{ga}}(t)+r_{\mathrm{L}} i_{\mathrm{ga}}(t)+v_{\mathrm{an}} \\
& v_{\mathrm{gb}}(t)=L \frac{\mathrm{d}}{\mathrm{d} t} i_{\mathrm{gb}}(t)+r_{\mathrm{L}} i_{\mathrm{gb}}(t)+v_{\mathrm{bn}} \\
& v_{\mathrm{gc}}(t)=L \frac{\mathrm{d}}{\mathrm{d} t} i_{\mathrm{gc}}(t)+r_{\mathrm{L}} i_{\mathrm{gc}}(t)+v_{\mathrm{cn}}
\end{aligned}
$$

A three-phase grid impedance can be emulated using a linear amplifier where the reference voltages are calcu-

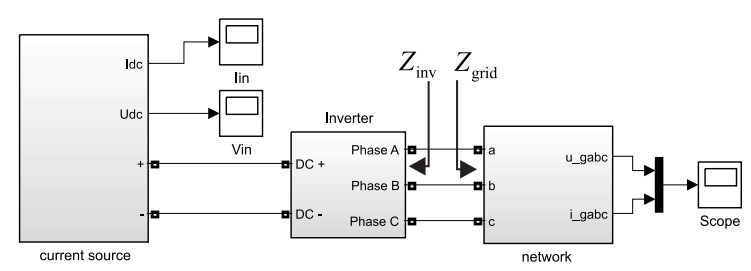

Figure 3. Overview of the grid-connected inverter.

lated based on the measured grid currents according to (1). This is an interesting idea since practically the amplifier could be configured to resemble an arbitrary grid impedance which is very attractive for stability studies of grid-connected inverters and for quality control of power converters.

A Simulink model according to to (1) was built to emulate the dynamics of grid impedance, as depicted in Figure 2. Moreover, a low-pass filter with a cut-off frequency of $10 \mathrm{kHz}$ was used to filter out switching ripple from the output of the derivative block. The value of grid inductance can be changed online which emulates the behavior of a real power grid. Moreover, the model could be easily modified to enable online variation of frequency, phase angle and resistance. However, the scope of this paper is limited to changing the value of inductance.

\section{Impedance-based instability studies}

The grid model was connected to a switching model of a three-phase inverter as illustrated in Figure 3. The model is built using the SimScape component library. The inverter is fed from a DC current source and connected to three-phase voltage sources. The reference values of grid voltages at the PCC were calculated according to (1). The grid currents become unstable when the ratio of inverter output impedance and the simulated grid impedance fail to satisfy the Nyquist stability criterion, i.e., when impedance q-components $Z_{\text {inv }}^{\mathrm{q}}$ and $Z_{\text {grid }}^{\mathrm{q}}$ form together an undamped resonance. Derivation of the inverter impedance model and discussing the stability criterion are out of the scope of this paper but the reader is urged to see (Messo et al., 2015) that shows how the Nyquist stability criterion can be applied to three-phase impedances.

Figure 4 shows the impedance ratio $Z_{\text {grid }}^{\mathrm{q}} / Z_{\text {inv }}^{\mathrm{q}}$ on the complex-plane when grid inductance is selected as $2 \mathrm{mH}$ and increased to $5 \mathrm{mH}$. The Nyquist stability criterion states that the system is unstable when the impedance ratio encircles the point $(-1,0)$. Thus, the system is stable when grid inductance is $2 \mathrm{mH}$ but becomes unstable when the inductance is increased to $5 \mathrm{mH}$.

The "Inverter" block in Figure 3 includes the actual power stage, AC filter and the control system. The inverter utilizes normal cascaded control scheme where DC voltage and AC currents are controlled to keep the DC voltage at a certain level and to feed power to the grid at unity power factor. The inverter utilizes a phase-locked-loop 


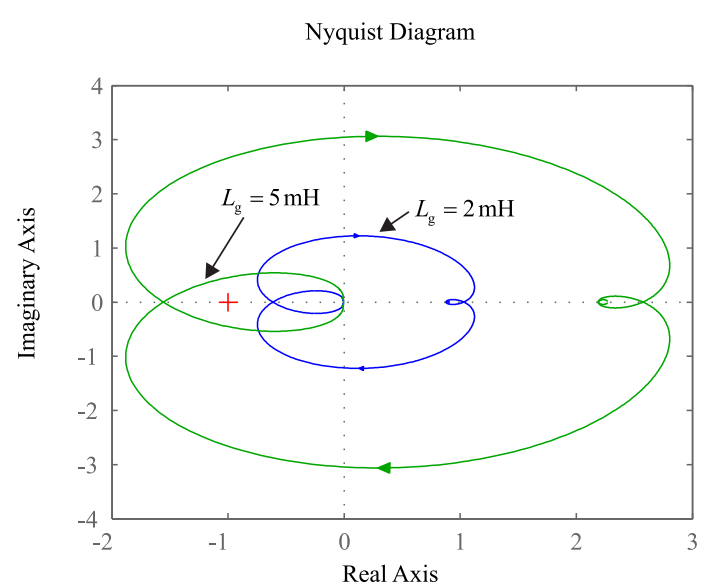

Figure 4. Ratio of inverter impedance and grid impedance on the complex-plane.

(PLL) to synchronize its output currents to grid voltages. The power stage and necessary measurements are built using the components found in the SimScape-toolbox as illustrated in Figure 6.

Phase-locked-loop makes the q-component of inverter output impedance resemble a negative resistance which can destabilize the inverter in a weak grid (Messo et al., 2013). The PLL was tuned to have a crossover frequency of $200 \mathrm{~Hz}$ which causes instability when grid inductance increases to $5 \mathrm{mH}$. Figure 5 shows simulated grid currents when the grid inductance is suddenly increased from 2 to $5 \mathrm{mH}$ at $0.5 \mathrm{~s}$ and the inverter becomes unstable due to high-bandwidth PLL. The simulator could be constructed by adding grid impedance in the model as inductive components and connecting an extra $3 \mathrm{mH}$ inductance at $0.5 \mathrm{~s}$ by using an ideal switch. However, in this case the simulation time is considerably longer. Therefore, using the proposed impedance model as in Figure 2 also enables smaller simulation time.

\section{Practical implementation}

The feasibility of grid impedance emulation method in a power-hardware-in-the-loop environment was tested by implementing the impedance model using a dSPACE real-time simulator and a three-phase linear amplifier PAS15000 manufactured by Spitzenberger. Photograph of the laboratory setup is as shown in Figure 7. The setup consists of a three-phase IGBT inverter supplied by a PV simulator feeding the three-phase linear amplifier through an isolation transformer.

The Simulink model for emulating inductive grid impedance is as shown in Figure 8. Ideal grid voltages were generated by three sine-wave sources phase shifted by 120 degrees. Voltage drop over the emulated phase inductance was obtained by multiplying the inductor current derivative by the desired inductance value. The output signal of the product block had to be low-pass filtered to avoid destabilizing the linear amplifier which is due to

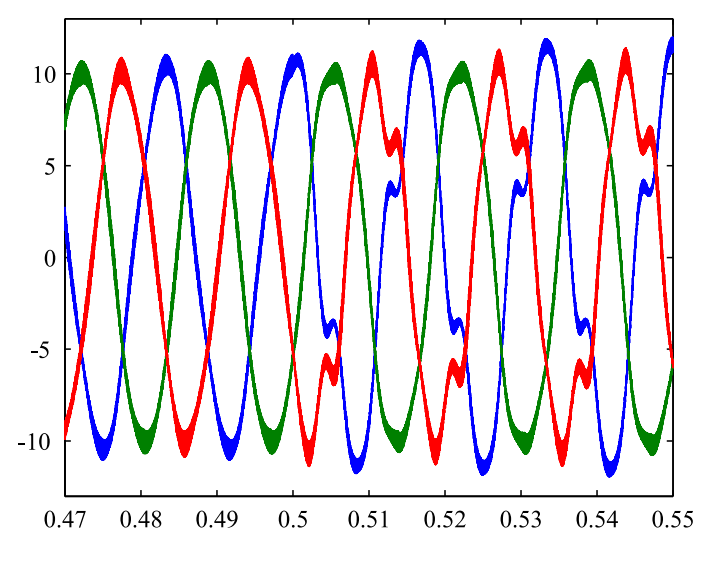

Figure 5. Grid currents become unstable due to increase in grid inductance from 2 to $5 \mathrm{mH}$ at $0.5 \mathrm{~s}$.

sampling delay of dSPACE. The impedance model was running on the same dSPACE-platform as the inverter control system. Therefore, the sampling frequency has to be set the same as inverter switching frequency $(8-12 \mathrm{kHz})$.

Input impedance of the linear amplifier, i.e., the emulated grid impedance was measured in the dq-domain using the inverter as a perturbation source and by measuring the frequency response from grid current q-component to grid voltage q-component. The grid impedance qcomponent is the ratio of these components as given in (2). The measurement setup is as depicted in Figure 9. PRBSinjection was added in the reference value of inverter output current q-component. The grid current and voltage qcomponents were measured and the grid impedance was computed using the methods discussed in (Roinila et al., 2015). The currents and voltage were measured in the dqreference frame tied to the inverter control system, i.e., by utilizing the grid voltage angle estimated by the PLL. The PLL crossover frequency was set to $2 \mathrm{~Hz}$ to avoid PLL from affecting the measured impedance at low frequencies.

$$
Z_{\mathrm{g}}^{\mathrm{q}}=\frac{\hat{v}_{\mathrm{oq}}}{\hat{i}_{\mathrm{oq}}}
$$

The isolation transformer has some resistive losses and stray inductance which have to be measured first to evaluate which part of the grid impedance is caused by the transformer and which is realized by the impedance emulator. The grid impedance q-component was measured while the value of emulated inductance value was set to zero. The measured and fitted impedances are shown in Figure 10. The isolation transformer has resistance of $400 \mathrm{~m} \Omega$ and inductance of approximately $600 \mu$ H. I.e., the phase is around 0 degrees at low frequencies with a constant magnitude and increases to 90 degrees at higher frequencies with magnitude increasing $20 \mathrm{~dB}$ per decade. It can be concluded that the impedance measurement is 


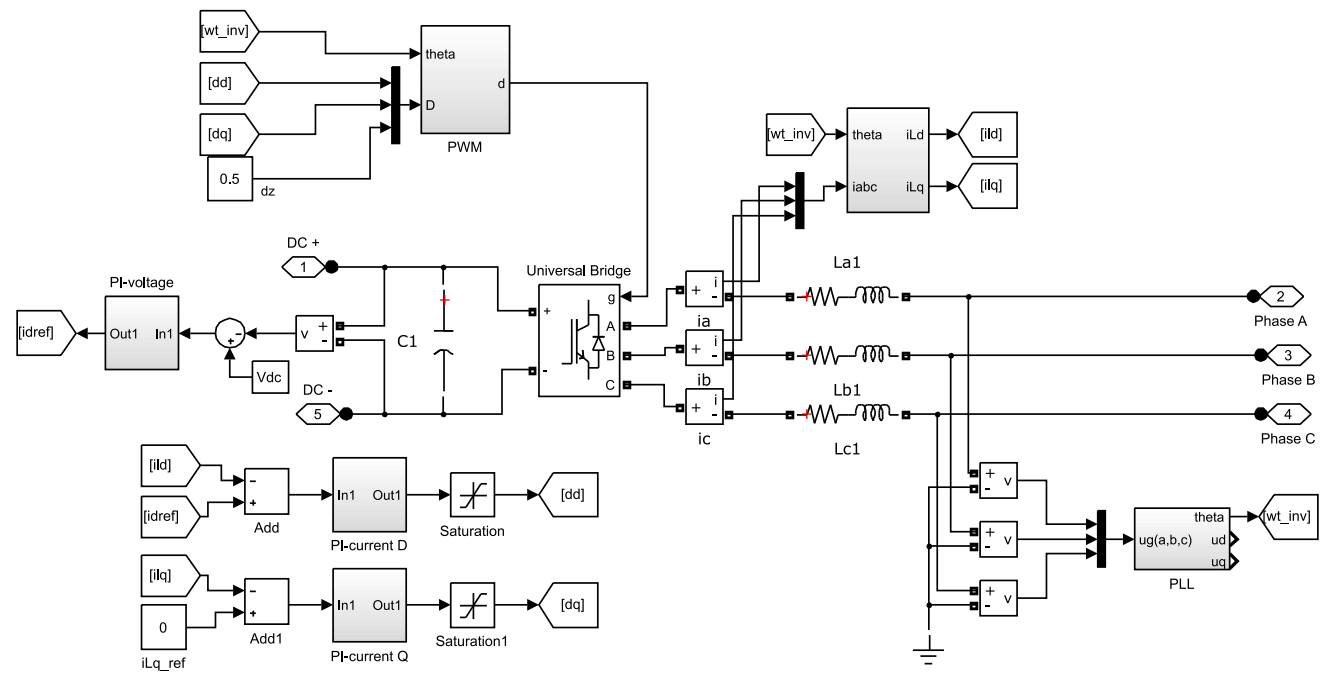

Figure 6. Inverter power stage with control system in Simulink.

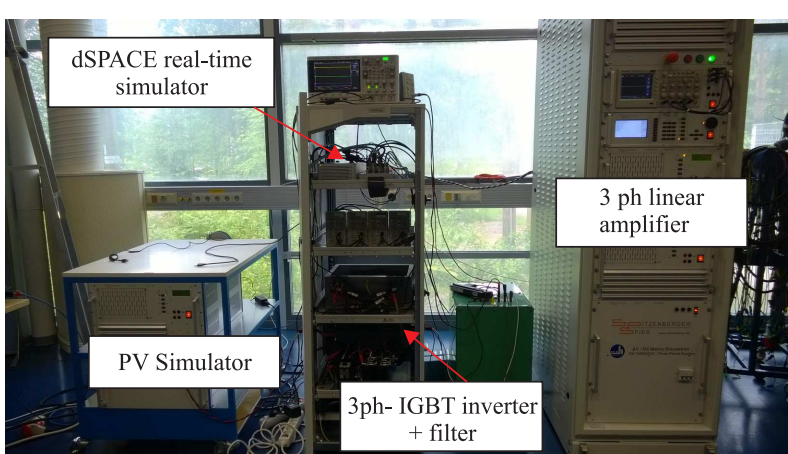

Figure 7. Three-phase inverter connected to a three-phase grid emulator.

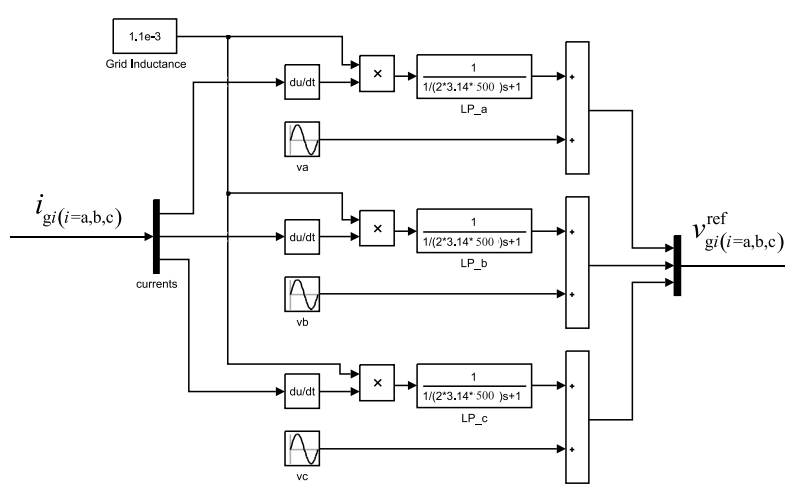

Figure 8. Impedance model implemented using the dSPACE real-time simulator.

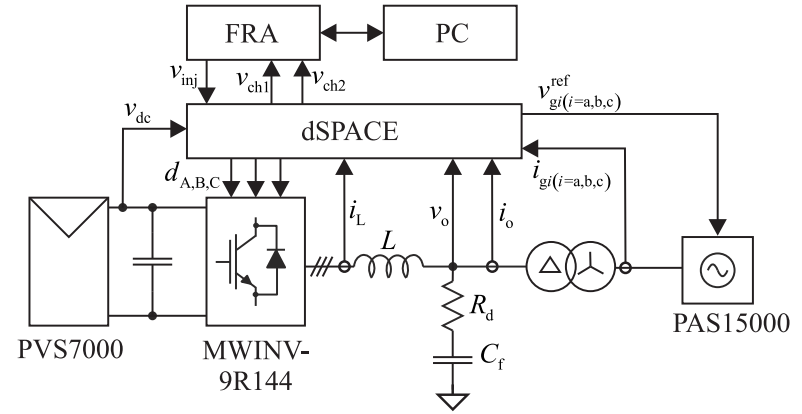

Figure 9. Setup for measuring the emulated grid impedance.

accurate up to few kilohertz after which the phase curve starts to drop due to sampling delay.

Figure 11 shows the measured grid impedance in green when the impedance emulator is activated. The inductance value was set to $1.1 \mathrm{mH}$ and the low-pass filter was tuned to have a crossover frequency of $500 \mathrm{~Hz}$ allowing stable operation of the linear amplifier. The red dots illustrate the impedance of a series RL-circuit with resistance equal to $400 \mathrm{~m} \Omega$ and inductance of $1.7 \mathrm{mH}$, i.e., the reference grid impedance includes the effect of isolation transformer. The magnitude of the emulated impedance follows the reference curve up to $400 \mathrm{~Hz}$ after which the impedance experiences an additional series resonance. Moreover, the phase starts to deviate from the reference value already around $50 \mathrm{~Hz}$. The inaccuracy of emulated impedance is caused by the low-pass filter which was required for stable operation. A low-pass filter with a single pole starts decreasing the phase of the impedance already one decade below cut-off frequency, i.e., at $50 \mathrm{~Hz}$. However, the low-pass filter is required for stability and cannot be set to higher frequency while sampling frequency is tied to inverter switching frequency. Reference of the grid impedance which includes the effect of transformer 

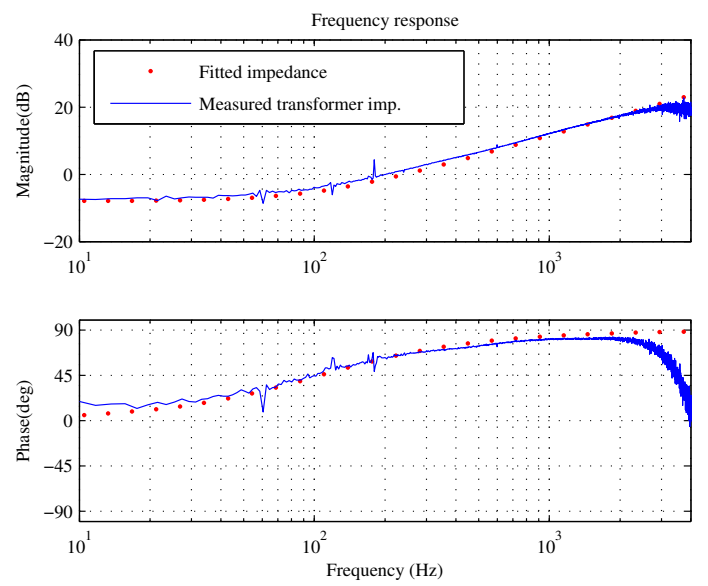

Figure 10. Measured transformer impedance q-component.
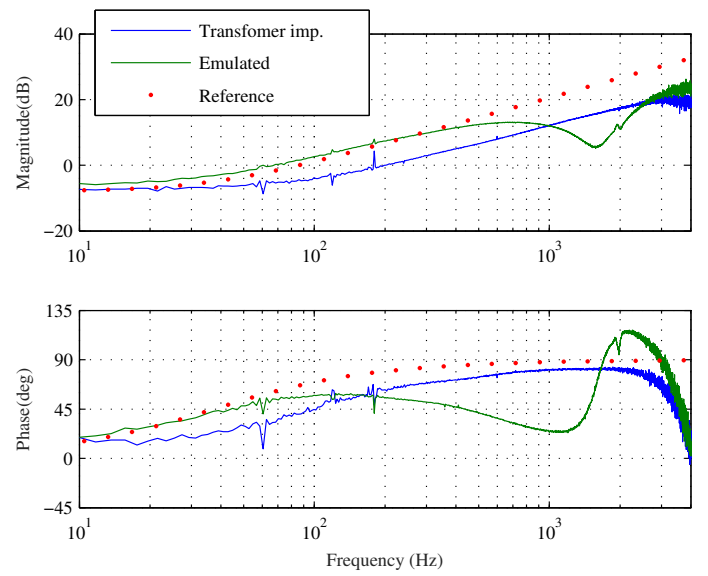

Figure 11. Measured grid impedance when impedance emulator is activated.

impedance is given as a function of frequency in (3).

$$
Z_{\mathrm{g}}^{\mathrm{q}}(j \omega)=0.4+j \omega 1.7 \cdot 10^{-3}
$$

Switching frequency of the inverter was increased to $12 \mathrm{kHz}$ and, therefore, the sampling frequency of the grid impedance emulator was increased as well. The sampling delay was effectively reduced by a factor of 1.5. The higher sampling frequency allows stable operation of the linear amplifier with emulated inductance value of 900 $\mu \mathrm{H}$ when the low-pass filter was tuned to have cut-off frequency of $1 \mathrm{kHz}$. Using higher value of emulated inductance value or increasing the cut-off frequency would destabilize the linear amplifier. This should be avoided since very large high frequency currents would flow in the circuit. (Enough to trip current sensor with 50 A current limit!)

Measured grid impedance and its reference value are shown in Figure 12. The magnitude curve follows the reference curve up to almost $1 \mathrm{kHz}$. However, the phase curve begins to deviate from reference curve approximately after $100 \mathrm{~Hz}$. This is expected since the low-pass
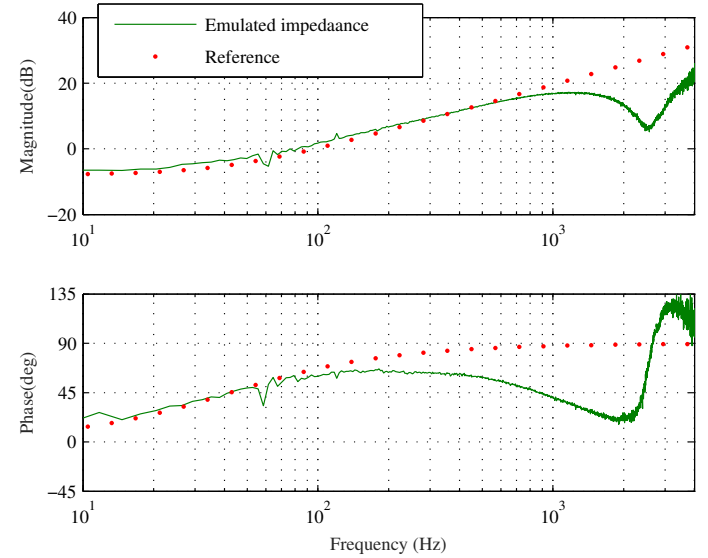

Figure 12. Measured grid impedance when impedance emulator is activated with higher sampling frequency.

filter has cut-off frequency of $1 \mathrm{kHz}$. It is not reasonable to increase the inverter switching frequency much higher since the IGBT switches require as much as $4 \mu$ s of blanking time to avoid shorting the DC capacitor. The reference curve for the emulated impedance is calculated according to (4).

$$
Z_{\mathrm{g}}^{\mathrm{q}}(j \omega)=0.4+j \omega 1.5 \cdot 10^{-3}
$$

Based on the measured impedances it can be concluded that the present impedance emulator is suitable for characterizing impedance-based instabilities occuring at lowfrequencies in cases when grid inductance has a maximum value around $1 \mathrm{mH}$. The limitation arises from the low sampling frequency which causes the phase of emulated impedance to deviate from the reference value at frequencies higher than $100 \mathrm{~Hz}$. Impedance-based interactions occurring at higher frequencies cannot be, therefore, reproduced. As a future research the sampling frequency of impedance emulator should be decoupled from the inverter control system which requires another realtime simulator for implementation.

\section{Conclusions}

The amount of grid-connected power electronic converters is increasing due to reduced price of renewable energy, such as wind and solar. At the same time stability of power grids is challenged by the inverter control functions, such as grid-synchronization algorithms. Evaluation methods to characterize stability issues introduced by grid-connected converters need to be developed to enable large-scale utilization of renewable energy.

The converter becomes unstable when its control system starts interacting with grid impedance which usually varies over time. In determining impedance-based stability, a large set of physical components is required in the laboratory to realize the variation in grid impedance. Moreover, contactors are required to change the grid 
impedance to emulate load changes in the grid which complicates the test setup and increases cost.

This paper studies a grid impedance emulation method using a dSPACE real-time simulator and three-phase linear amplifier which eliminates the need for bulky and expensive passive components. In the proposed method grid impedance is modeled inside the real-time simulator which allows changing the value of grid impedance online. Performance of the impedance emulation method is studied by measuring the input impedance of the impedance-emulator by using a three-phase IGBT inverter to generate the small-signal excitation in its output currents. The impedance emulator is shown to be very sensitive to sampling delay which degrades the phase behavior of the emulated grid impedance. Moreover, the delay easily destabilizes the linear amplifier when large grid inductance is to be emulated. This issue should be treated with caution since very large high-frequency currents can flow in the circuit. Future work will include implementing the grid impedance emulation method by an additional realtime simulator in order to achieve higher sampling frequency and smaller delay.

\section{Aknowledgements}

This research was supported by the Academy of Finland.

\section{References}

P. Belkin. Event of 10-22-09. CREZ Technical Conference, Electrical Reliability Council of Texas, 2010. URL www. ercot. com.

B. K. Bose. Global energy scenario and impact of power electronics in 21st century. IEEE Trans. Ind. Electron., 60(7): 2638-2651, 2013. doi:10.1109/TIE.2012.2203771.

J. H. R. Enslin and P. J. M. Heskes. Harmonic interaction between a large number of distributed power inverters and the distribution network. IEEE Trans. Power Electron., 19(6): 1586-1593, 2004. doi:10.1109/TPEL.2004.836615.

A. Gavrilovic. Ac/dc system strength as indicated by short circuit ratios. International Conference on $A C$ and DC Power Transmission, pages 27-32, 1991.

L. Harnefors, X. Wang, A. Yepes, and F. Blaabjerg. Passivity-based stability assessment of grid-connected vscs - an overview. IEEE Journal of Emerging and Selected Topics in Power Electronics, 4(1):116-125, 2016. doi:10.1109/JESTPE.2015.2490549.

L. Jessen, F. W. Fuchs, and C. Kiel. Modeling of inverter output impedance for stability analysis in combination with measured grid impedances. IEEE 6th Int. Symp. Power Electron. Distrib. Gener. Syst. (PEDG), pages 1-7, 2015. doi:10.1109/PEDG.2015.7223037.

P. Kotsampopoulos, F. Lehfuss, G. Lauss, B. Bletterie, and N. Hatziargyriou. The limitations of digital simulation and the advantages of phil testing in studying distributed generation provision of ancillary services. IEEE Trans. Ind. Electron., 62(9):5502-5515, 2015. doi:10.1109/TIE.2015.2414899.
T. Messo, J. Jokipii, A. Mäkinen, and T. Suntio. Modeling the grid synchronization induced negative-resistor-like behavior in the output impedance of a three-phase photovoltaic inverter. 4th IEEE International Symposium on Power Electronics for Distributed Generation Systems (PEDG), pages 1-7, 2013. doi:10.1109/PEDG.2013.6785602.

T. Messo, A. Aapro, and T. Suntio. Generalized multivariable small-signal model of three-phase grid-connected inverter in dq-domain. IEEE 16th Workshop on Control and Modeling for Power Electronics (COMPEL), pages 1-8, 2015. doi:10.1109/COMPEL.2015.7236460.

T. Roinila, T. Messo, T. Suntio, and M. Vilkko. Pseudo-random sequences in DQ-domain analysis of feedforward control in grid-connected inverters. IFAC-PapersOnLine, 48(28):13011306, 2015. doi:10.1016/j.ifacol.2015.12.311.

J. Sun. Impedance-based stability criterion for grid-connected inverters. IEEE Trans. Power Electron., 26(11):3075-3078, 2011. doi:10.1109/TPEL.2011.2136439.

Teuvo Suntio, Tuomas Messo, and Joonas Puukko. Power Electronic Converters: Dynamics and Control in Conventional and Renewable Energy Applications. Wiley VCH, 2017. ISBN 978-3-527-34022-4.

X. Wang, F. Blaabjerg, and W. Wu. Modeling and analysis of harmonic stability in an ac power-electronics-based power system. IEEE Trans. Power Electron., 29(12):6421-6432, 2014. doi:10.1109/TPEL.2014.2306432.

B. Wen, D. Boroyevich, R. Burgos, P. Mattavelli, and Z. Shen. Analysis of d-q small-signal impedance of grid-tied inverters. IEEE Trans. Power Electron., 31(1):675-687, 2016. doi:10.1109/TPEL.2015.2398192.

D. Yang, X. Ruan, and H. Wu. Impedance shaping of the gridconnected inverter with lcl filter to improve its adaptability to the weak grid condition. IEEE Trans. Power Electron., 29 (11):5795-5805, 2014. doi:10.1109/TPEL.2014.2300235. 